\title{
A PARALISIA CEREBRAL NA ADOLESCÊNCIA: RESULTADOS DE UMA INVESTIGAÇÃO
}

\author{
Ana Paula Matos \\ Joana Castela Lobo
}

Resumo: Revela-se de primordial importância estudar o autoconceito, o coping e a saúde mental de adolescentes com Paralisa Cerebral. Essa população tem sido muito pouco estudada. Além disso, essas variáveis podem contribuir para a adaptação desses jovens que se confrontam com situações mais adversas, podendo ser fatores protetores e de risco da sua saúde psicológica. Estudamos uma amostra de 78 sujeitos com Paralisia Cerebral (idades entre os 12 e os 19 anos). Encontramos os seguintes resultados: a) as moças apresentam valores de psicopatologia mais elevados do que os rapazes; b) à medida que aumenta a idade diminuem os valores do autoconceito e aumentam os do "coping não ativo"; c) alguns tipos de diagnóstico de paralisia cerebral diferenciam-se pelo autoconceito; d) o autoconceito correlaciona-se negativamente com todos os índices de psicopatologia; e) o "coping não ativo" correlaciona-se negativamente com a ansiedade e com alguns índices de sintomatologia psicopatológica do BSI.

Palavras-chave: Adolescência. Paralisia cerebral. Coping. Autoconceito. Psicopatologia.

\section{Introdução}

Com o evoluir da ciência tem sido possível manter vidas e recuperá-las de situações que, noutros tempos, tinham como destino a morte. Contudo, nem sempre a qualidade de vida é a desejável (Thompson \& Raezer, 1998). 
A doença crônica afeta, assim, um elevado número de crianças e famílias, representando um indutor de stress permanente, com riscos de dificuldades no ajustamento. A Paralisia Cerebral é um desses exemplos, e o que vamos considerar.

A Paralisia Cerebral é considerada por alguns autores como a doença física neurológica congênita mais comum (Florian \& Findler, 2001). Os seus sinais característicos incluem espasticidade, perturbações do movimento, fraqueza muscular, ataxia e rigidez; e as manifestações que lhe estão associadas dependem da magnitude, extensão e localização das lesões do Sistema Nervoso Central (SNC) (Koman, Smith, \& Shilt, 2004).

A Paralisia Cerebral representa então uma constelação de perturbações que apresentam várias características em comum (Alexander, 1990):

a) há uma lesão no SNC, que afeta o controle motor;

b) ocorre numa fase intrauterina, perinatal ou nos primeiros anos de vida;

c) não é progressiva.

Pensamos ser de primordial importância estudar o autoconceito, o coping e a saúde mental de adolescentes com Paralisa Cerebral. Essa população tem sido muito pouco estudada. E, mesmo na adolescência de indivíduos normais, comparada com outros estágios de desenvolvimento, ocorrem enormes mudanças e desafios, e os jovens são confrontados com muitas situações para as quais não estavam preparados. O processo de coping é, então, particularmente importante durante a adolescência.

Os adolescentes com Paralisia Cerebral confrontam-se, muito provavelmente, com desafios e dificuldades ainda maiores (as tarefas desenvolvimentais normais, acrescidas com os obstáculos que a doença lhes impõe).

Segundo Seiffge-Krenke (1998), nos adolescentes com doença crônica encontramos associadas limitações e dificuldades em áreas tão importantes para o seu desenvolvimento como a vida acadêmica ou profissional (absentismo mais frequente, rendimento mais pobre), as relações sociais (maior dependência e isolamento social, interações sociais mais restritas, menos relações próximas com os seus pares sociais, menos amigos, menos relações de namoro, relacionamento sexual mais tardio) e a sua própria imagem física.

Tudo isso pode contribuir para um autoconceito mais pobre nesses adolescentes. Mann, Hosman, Schaalma e Vries (2004) e Sukumaran,Vickers, Yates e Garralda (2003) salientaram que a autoestima e o autoconceito podem ser fatores protetores e de risco na doença física e psicológica.

A forma como os jovens lidam e se adaptam às situações indutoras de stress pode influenciar a sua adaptação.

A literatura tem mostrado que adolescentes com doença crônica estão em maior risco de desenvolver problemas comportamentais e emocionais (Seiffge-Krenke, 1998). 
Wallander (1988, 1989 citado por Bradford, 1997) comprovaram com os seus estudos que os doentes crônicos são uma população de risco para problemas psicológicos, e que manifestavam níveis mais elevados de problemas sociais e de comportamento, quando comparados com uma amostra controle de indivíduos saudáveis. Outros autores, como Salmon (2000) e Joyce-Moniz e Barros (2005), têm salientado a existência de emocionalidade negativa (por exemplo, ansiedade, depressão e cólera) associada à doença física.

Pless e Roghmann (1971, citados por Thompson \& Raezer, 1998), através de estudos epidemiológicos, concluíram que crianças com problemas médicos crônicos apresentavam uma proporção acrescida de problemas emocionais e sociais, quando comparadas com crianças saudáveis. Em 1987, Cadman (citado por Thompson \& Raezer, 1998) confirmaram que $33 \%$ das crianças com doença crônica tinham, também, pelo menos um diagnóstico de perturbação da DSM-III. Pless e Stein (1996) concluíram que os estudos têm mostrado consistentemente que o risco para desenvolver problemas emocionais secundários é significativamente maior em crianças com doença crônica do que em crianças saudáveis.

Segundo Harris (1998) e Miller e Clark (1998), é mais provável encontrar, na população com Paralisia Cerebral, distúrbios emocionais e do comportamento.

A combinação das exigências que se colocam ao adolescente com os recursos que este tem disponíveis vai certamente afetar a sua saúde mental. No que diz respeito aos recursos psicológicos, pensamos que o coping e o autoconceito podem ser vistos como importantes fatores protetores que podem manter a resiliência dos adolescentes expostos a essas situações adversas.

Livneh e Antonak (2005) salientaram também a importância do stress, do coping e do autoconceito na adaptação psicossocial à doença e à incapacidade crônicas. Koman, Smith e Shilt (2004) apontam a necessidade de se melhorar a autoestima de doentes com Paralisia Cerebral.

Dentre os vários modelos existentes de stress e coping, a maior parte deles compreensivos e integradores e muitos baseando-se no modelo de Lazarus e Folkman (1984), salientamos dois: o modelo de Wallander, Varni, Babani, Banis e Wilcox (1989), que desenvolve a ideia de variabilidade no ajustamento, individual e familiar, à doença crônica e à deficiência, como função da interdependência de fatores de risco e de resiliência e da forma como eles atuam. É um modelo multifatorial que salienta, entre outros aspectos, a importância das aptidões de coping e do autoconceito, para a adaptação; e o modelo de Thompson e Gustafson (1996) é um modelo que coloca a hipótese de que a relação entre a doença e o resultado da adaptação é uma função de transações entre aspectos biomédicos (que refletem o tipo e a gravidade da doença), aspectos demográficos (como a idade, o sexo e o nível socioeconômico) e processos psicossociais (que incluem a 
avaliação da doença e do stress do dia a dia, as expectativas de eficácia, o locus de controle, os estilos de coping e o funcionamento familiar).

São ambos modelos complexos, que se sobrepõem em alguns aspectos e se complementam noutros. Serviram-nos de quadro de referência para a presente investigação, tendo nós optado por selecionar algumas das suas variáveis mais importantes (uma vez que se torna difícil testar qualquer um dos modelos, num único estudo) e estudá-las numa amostra de adolescentes com Paralisia Cerebral.

Nesse estudo, tivemos essencialmente dois grandes objectivos gerais.

Primeiro, quisemos conhecer melhor a distribuição de variáveis sociodemográficas (gênero, idade, nível socioeconômico) e clínicas (gravidade de patologia motora e tipo de diagnóstico clínico) num grupo de adolescentes com Paralisia Cerebral e a relação dessas variáveis com construtos psicológicos como o autoconceito, o coping e a presença de sintomatologia psicopatológica.

Segundo, quisemos investigar se o autoconceito e as estratégias de coping estavam relacionados com a vulnerabilidade para desenvolver sintomatologia psicopatológica em adolescentes com Paralisia Cerebral.

Formulamos as seguintes hipóteses principais:

Hipótese 1: As variáveis sociodemográficas Gênero, Idade e Estatuto Socioeconômico associam-se de forma significativa com as variáveis psicológicas Autoconceito, Coping e Sintomatologia Psicopatológica (ansiedade, depressão e outros sintomas psicopatológicos avaliados pelo BSI).

Hipótese 2: O Tipo Clínico de Paralisia Cerebral associa-se de forma significativa com as variáveis psicológicas Autoconceito, Coping e Sintomatologia Psicopatológica (ansiedade, depressão e outros sintomas psicopatológicos avaliados pelo $\mathrm{BSI}$ ).

Hipótese 3: A gravidade da Patologia Motora na Paralisia Cerebral relaciona-se de uma forma significativa e negativa com o Autoconceito e com as Estratégias de Coping e de uma forma significativa e positiva com a Sintomatologia Psicopatológica (depressão, ansiedade e outra sintomatologia psicopatológica avaliada pelo BSI).

Hipótese 4:Valores mais baixos de Autoconceito e estratégias de Coping mais pobres relacionam-se de uma forma significativa com valores mais elevados de Sintomatologia Psicopatológica (depressã, ansiedade e outra sintomatologia psicopatológica avaliada pelo BSI).

O recolhimento da amostra efetuou-se nos Centros de Reabilitação de Paralisia Cerebral de Coimbra e do Porto, após ter sido autorizado o estudo pelas respectivas instituições. A amostra do presente estudo é composta por 78 indivíduos com Paralisia Cerebral, com idades entre os 12 e 19 anos, que colaboraram no estudo de uma forma informada, voluntária e anônima. Dos 78 indivíduos com Paralisia Cerebral, 45 são do sexo masculino e 33 pertencem ao sexo feminino. A média de idades é de 16,2 anos. 
Colocou-se como critério de inclusão ter uma capacidade intelectual normal, para poder compreender as questões e os construtos subjacentes a um conjunto de questionários que usamos para avaliar as variáveis em estudo e que questionam diretamente o adolescente com Paralisia Cerebral. Pretendíamos saber o que o próprio adolescente, e não os seus pais ou técnicos de saúde, pensava sobre questões importantes relativas a si (o seu autoconceito, as suas estratégias de coping) e à forma como se sente (sintomatologia depressiva, ansiosa e outros sintomas psicopatológicos em geral).

Relativamente à variável nível socioeconômico, recorreu-se à classificação de Sedas Nunes e Miranda (1970), que estratifica os indivíduos em quatro camadas sociais, tendo em conta o grau de instrução e a profissão dos pais. Nessa estratificação, o nível IV é considerado o mais baixo e o nível I o mais elevado.

O grupo socioeconômico mais frequente na nossa amostra é a camada social IV, com 46,2\%, seguida da III, com $34,6 \%$. As camadas II e I têm, respectivamente, $15,4 \%$ e $3,8 \%$.

Nessa amostra, os quadros clínicos espásticos são, sem dúvida, os mais frequentes. Em primeiro lugar aparece o tipo espástico hemiparético, com $43,6 \%$, seguido do espástico diplégico, com $24,4 \%$, e depois do espástico tetraparético, com $16,7 \%$. O tipo disquinético aparece em $14,1 \%$ da amostra e o atáxico, minoritariamente, em $1,3 \%$.

Utilizamos um conjunto de questionários de autoavaliação, de tipo Likert:

a) Children's Depression Inventory (CDI, Kovacs, 1983). Utilizamos a versão portuguesa de Simões (1999);

b) Revised Children's Manifest Anxiety Scale (RCMAS, Reynolds \& Richmond, 1978). Usamos a versão portuguesa de Fonseca (1992). Essa escala tem uma nota global e duas dimensões:"ansiedade global" e "mentira / desejabilidade social";

c) Brief Symptom Inventory (BSI, Derogatis, 1982). Utilizamos a versão portuguesa de Canavarro (1999). Esse inventário tem três índices gerais:Índice Geral de Sintomas (IGS), Total de Sintomas Positivos (TSP) e Índice de Sintomas Positivos (ISP); tem também nove dimensões: "somatização", "obsessões-compulsões", "sensibilidade interpessoal", "depressão", "ansiedade", "hostilidade", "ansiedade fóbica", "ideação paranóide" e "psicoticismo".

d) Piers-Harris Self-Concept Questionnaire (PHSCS, Piers, 1984). Utilizamos a versão portuguesa, que foi traduzida por Simões e Albuquerque (1989) e foi adaptada e estudada psicometricamente por Veiga (1989). Esse questionário tem uma nota global e seis dimensões: "aspecto comporta- 


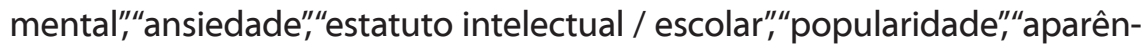
cia / atributos físicos" e"satisfação / felicidade".

e) Coping Across Situations Questionnaire (CASQ, Seiffge-Krenke \& Shulman, 1990). Usamos a versão portuguesa de Cleto (1998). Esse questionário tem dois fatores:"coping ativo" e "coping não ativo".

A Escala de Inteligência de Wechsler para crianças (WISC, versão portuguesa de Marques, 1970) foi por nós utilizada para decidir da inclusão do sujeito na amostra, uma vez que só incluímos indivíduos com nível intelectual normal.

O grau de compromisso motor na Paralisia Cerebral foi avaliado recorrendo ao Gross Motor Function Classification System for Cerebral Palsy (Palisano et al., 1997).

\section{Resultados}

Estudo da relação entre as variáveis sociodemográficas e as variáveis psicológicas (Autoconceito, Coping e Psicopatologia)

\section{Comparação entre gêneros}

Quisemos averiguar se, nesse grupo de adolescentes com Paralisia Cerebral, a diferença entre os sexos era significativa para as variáveis psicológicas avaliadas: Autoconceito, Coping, Depressão, Ansiedade e outros Sintomas Psicopatológicos.

Para esse efeito, utilizamos o teste $t$ de Student que pressupõe que as variáveis tenham uma distribuição normal.

Começamos por testar a Aderência à Normalidade através do teste Kolmogorov-Smirnov para todas as variáveis psicológicas. Com esse teste verificamos que apenas apresentam uma distribuição normal a ansiedade total e a "ansiedade global", avaliadas pelo RCMAS, e o total de sintomas positivos do BSI e a respectiva dimensão"obsessões-compulsões".Contudo, uma vez que a nossa amostra é composta por mais de 30 indivíduos, pudemos utilizar a estatística paramétrica para a análise dos resultados.

Verificaram-se diferenças estatisticamente significativas entre sexos para o fator "ansiedade" da escala de autoconceito ( $T=-2,831 ; p=0,006 ; M=4,88$ vs $M=6,44)$, para o total de ansiedade $(T=2,013 ; p=0,048 ; M=19,21$ vs $M=16,80)$ e para a "ansiedade global" ( $T=2,077 ; p=0,041 ; M=5,76$ vs $M=4,11)$, e, também, para os índices IGS ( $T=2,262 ; p=0,027 ; M=1,07$ vs $M=0,80)$ e ISP $(T=2,085 ; p=0,040$; $M=1,8$ vs $M=1,64$ ) do $B S I$. Quanto às dimensões avaliadas pelo $B S I$, encontramos diferenças significativas para a "sensibilidade interpessoal" ( $T=3,120 ; p=0,003$; $M=6,09$ vs $M=3,73)$, para a "depressão" ( $T=2,810 ; p=0,007 ; M=7,64$ vs $M=4,20)$ e para o"psicoticismo" ( $\mathrm{T}=2.091 ; \mathrm{p}=0,040 ; \mathrm{M}=5,21$ vs $\mathrm{M}=3,53)$. 
É o sexo feminino que apresenta valores mais elevados em todos esses índices.

\section{Relação das variáveis psicológicas com a idade}

Quisemos estudar, também, a possível influência da variável idade nas variáveis psicológicas avaliadas pelos questionários de autorresposta. Para esse efeito, procedemos à determinação do coeficiente de correlação de Spearman, e analisamos o seu nível de significância. Optamos por esse teste de estatística não paramétrica devido ao fato de, como já referimos, a nossa amostra não ter uma distribuição normal em muitas das variáveis psicológicas estudadas.

Verificamos que existe uma correlação positiva e significativa entre a idade e o "coping ativo" ( $r=0,349 ; p=0,002)$, o que indica que, nesses jovens com Paralisia Cerebral, suas estratégias ativas de lidar com as situaçõesproblema vão aumentando com a idade.

Obtivemos ainda correlações significativas, mas negativas, entre a idade e alguns fatores do autoconceito: "aspecto comportamental ( $r=-$ $0,237 ; p=0,037)$, "ansiedade" ( $r=-0,241 ; p=0,036)$, "satisfação/felicidade" ( $r=-$ 0,$227 ; p=0,046$ ). O que significa que à medida que aumenta a idade, diminuem os valores do autoconceito.

\section{Relação das variáveis psicológicas com o nível socioeconômico}

Através da análise da variância simples (ANOVAs), averiguamos se existiam diferenças significativas entre os quatro níveis socioeconômicos nas variáveis psicológicas (Autoconceito, Coping e Psicopatologia) e não obtivemos nenhum valor estatisticamente significativo.

Podemos, dessa forma, concluir que os indivíduos das várias camadas sociais não se diferenciam nas variáveis psicológicas em estudo.

Estudo da relação entre as variáveis clínicas de Paralisia Cerebral e as variáveis psicológicas

\section{Níveis de compromisso motor}

Considerando os cinco níveis de compromisso motor avaliados pela equipe técnica que acompanha cada jovem, e obtidos através do Gross Motor Function Classification System for Cerebral Palsy (Palisano et al., 1997), tentamos estabelecer relações entre esses níveis e as variáveis autoconceito, coping, depressão, ansiedade e outra sintomatologia psicopatológica. Assim, efetuamos análises da variância simples (ANOVAs) e encontramos que o único valor estatisticamente significativo foi o correspondente à di- 
mensão"ansiedade" do BSI ( $\mathrm{F}=2,842 ; \mathrm{p}=0,030)$. No entanto, através do teste Post Hoc de Bonferroni verificamos que essa diferença entre as médias deixou de se revelar significativa.

Mesmo agrupando os níveis com menor número de sujeitos (ou seja, os níveis 3, 4 e 5) num mesmo nível, a análise da variância simples continuou a não revelar diferenças significativas entre os três grupos.

\section{Tipos clínicos de Paralisia Cerebral}

Fizemos um estudo semelhante para os tipos clínicos de Paralisia Cerebral. Através da análise da variância simples (ANOVAs) comparamos os vários grupos de diagnósticos:"espástico hemiparético";"espástico diplégico";"espástico tetraparético"; "disquinético"e eatáxico".

Nessa análise, pareceu-nos mais adequado retirar um indivíduo que era o único com o tipo atáxico de Paralisia Cerebral.Por essa razão, o número de sujeitos, para essa análise, foi de 77.

Encontramos valores significativos para o total de autoconceito $(F=4,153 ; p=0,009)$ e para alguns dos seus fatores:"aspecto comportamental" $(F=2,966 ; p=0,038)$ e "estatuto intelectual / escolar" $(F=3,998 ; p=0,011)$.

Na sequência desses resultados analisamos os valores das respectivas médias.

A realização do teste Post Hoc Bonferroni, em conjunto com a análise das médias, permite-nos concluir que tanto no total do autoconceito ( $p=0,011 ; M=47$ vs $M=56,18$ ) como no fator "estatuto intelectual / escolar" $(p=0,008 ; M=7,68$ vs $M=11,27$ ), as diferenças que se encontram entre $o$ grupo "espástico hemiparético" e o "disquinético" são significativas, apresentando esse último grupo médias mais elevadas. Quanto ao valor de $\mathrm{F}$ significativo encontrado na análise da variância para o "aspecto comportamental", essa diferença não se manteve significativa no teste Post Hoc de Bonferroni.

\section{Estudo das relações entre as variáveis psicológicas}

\section{Análise da relação entre o Autoconceito e a depressão, a ansiedade e a sintomatologia psicopatológica}

Finalmente, e relativamente ao estudo de uma possível relação entre os valores obtidos no questionário de Autoconceito e os resultados obtidos nos questionários que avaliam a depressão, a ansiedade e a sintomatologia psicopatológica geral (BSI), efetuamos coeficientes de correlação de Spearman. 
Como esperávamos, a associação entre os índices do autoconceito (nota global e respectivos fatores) e as variáveis depressão e ansiedade revelou-se, no geral, negativa e significativa (correlações do autoconceito com a depressão, de -,275, $\mathrm{p}<.05 \mathrm{a}-, 506, \mathrm{p}<.01$; correlações do autoconceito com a ansiedade de $-, 243, \mathrm{p}<.05 \mathrm{a}-, 437, \mathrm{p}<.01)$.

No entanto, na dimensão "aspecto comportamental", essa correlação é positiva e significativa $(r=0,354$ e $p=0,002)$. Essa relação justifica-se pelo significado que tem o fator "mentira", que é um fator de "desejabilidade social." Ou seja, a imagem favorável que se pretende passar aos outros apresenta uma relação significativa com o fator "aspecto comportamental" do autoconceito (enquanto percepção que o sujeito tem do seu comportamento e grau de responsabilidade que assume pelas suas ações).

Quanto às relações que se estabelecem entre os índices do autoconceito e os índices de psicopatologia geral do BSI, obtivemos, também, correlações negativas e significativas.

Duma forma geral, podemos dizer que o autoconceito mantém com os índices globais (IGS de $r=-, 381, p<.01$ a $r=-, 566, p<.01$; TSP de $r=-, 296$, $\mathrm{p}<.01$ a $\mathrm{r}=-, 532, \mathrm{p}<.01$; ISP de $\mathrm{r}=-, 267, \mathrm{p}<.50$ a $\mathrm{r}=-, 335, \mathrm{p}<.01$ ) e com as dimensões do BSI ("somatização", de $r=-, 250$ a r=-,408; "obsessões-compulsões, de $r=-, 244$ a r=-,448"; "sensibilidade interpessoal", de $r=-, 279$ a $r=-, 495$; "depressão", de r=-,332 a r=-,640;"ansiedade", de r=-,235 a r=-,476;"hostilidade",de r=-,248 a r=-,456;"ansiedade fóbica",de r=-,308 a r=-,426;"ideação paranóide", de r=-,259 a r=-,494 e psicoticismo", de r=-,228 a r=-,541) relações negativas significativas. Níveis mais baixos de autoconceito associam-se a níveis mais elevados de psicopatologia.

Análise da relação entre as estratégias de coping e a depressão, a ansiedade e outra sintomatologia psicopatológica

Quanto à variável estratégias de coping, esta foi analisada da mesma forma que o autoconceito. Executamos, também, correlações de Spearman entre os valores de coping (ativo e não ativo) e os valores de depressão, ansiedade e dos índices de psicopatologia do BSI.

As relações estabelecidas entre o"coping ativo" e os níveis de depressão e de ansiedade não são significativas.

No caso do "coping não ativo", a correlação com as medidas do questionário de ansiedade é negativa e significativa para a nota global de ansiedade $(r=-0,352 ; p=0,002)$ e para a "ansiedade global" $(r=-0,267 ; p=0,019)$.

Pudemos verificar que, na relação com as várias medidas do inventário de sintomas psicopatológicos (BSI), o "coping ativo" não revela associações significativas.

Já o "coping não ativo" estabelece relações negativas e significativas com o IGS ( $r=-0,237 ; p=0,038)$, com a dimensão "somatização" $(r=-0,285$; 
$p=0,020)$, com a "depressão" $(r=-0,280 ; p=0,014)$, com a "ansiedade" ( $r=-$ $0,233 ; p=0,042)$, com a "hostilidade" $(r=-0,229 ; p=0,045)$ e com o"psicoticis$m o "(r=-0,228 ; p=0,046)$.

Análise da relação entre os níveis de depressão, de ansiedade e de sintomatologia psicopatológica avaliada pelo BSI

Para não tornarmos o presente artigo mais extenso, decidimos não apresentar os resultados, mas apenas referir que efetuamos também correlações de Spearman entre as várias medidas de psicopatologia.Todas estas medidas (depressão avaliada pelo CDI, nota total de ansiedade avaliada pela RCMAS, e índices de sintomatologia psicopatológica avaliados pelo BSI) apresentam, entre si, correlações positivas, significativas. Apenas os fatores "ansiedade global" da RCMAS e "hostilidade" do BSI apresentaram relações positivas, mas não significativas, com as outras variáveis.

\section{Considerações finais}

Relativamente a variáveis sociodemográficas, como o gênero, o nosso grupo com Paralisia Cerebral é bem representativo dos dados que encontramos na bibliografia consultada. Na literatura, a prevalência de Paralisia Cerebral é superior no sexo masculino, sendo essa diferença de 57,8\% para 42,2\% (Andrada, 1986). Efetivamente, a amostra que recolhemos tem uma maior percentagem de indivíduos do sexo masculino e essa diferença faz-se sentir, praticamente, com os mesmos valores, ou seja, $57,7 \%$ do sexo masculino e $42,3 \%$ do sexo feminino.

Também, em relação à prevalência dos tipos clínicos, os dados apresentados por Andrada (1986) correspondem aos obtidos por nós. Realmente os síndromes dominantes são os espásticos, que aparecem na literatura como $85,5 \%$ dos casos e nos aparecem em $85 \%$ da amostra, seguidos pelos síndromes disquinéticos com 9,2\%, que aparecem na nossa amostra em $14 \%$ e, finalmente, os atáxicos que Andrada (1986) refere serem 4,1\% e, no nosso estudo, participam em apenas $1 \%$. Uma possível justificação para a baixa frequência no nosso estudo da ataxia talvez seja porque aos casos de ataxia aparece, muitas vezes, associado o atraso mental (Russman, 1991), e um dos critérios de inclusão no nosso grupo com Paralisia Cerebral era ter capacidade intelectual normal.

Verificamos também a existência de um predomínio dos níveis socioeconômicos mais baixos, no nosso grupo de adolescentes com Paralisia Cerebral. É preciso não esquecer que o recolhimento da amostra foi feita com usuários dos Centros de Reabilitação de Paralisia Cerebral, cujos usuários pertencem, sobretudo, às classes mais baixas. 
Em termos etiológicos, a Paralisia Cerebral pode afetar, indiferentemente, qualquer classe social. Mas vários investigadores têm feito referência a esse aspecto das doenças físicas crônicas aparecerem numa frequência acrescida em crianças com outros fatores de risco para o desajustamento, como seja a pobreza. O que a investigação ainda não concluiu foi se será a pobreza em si o fator de risco, se será algum outro fator associado a ela (Pless \& Stein, 1996).

No caso da nossa amostra com Paralisia Cerebral, não se verificou nenhuma relação significativa entre o nível socioeconômico e as variáveis psicológicas em estudo, pelo que não podemos atribuir os resultados encontrados nas mesmas à influência dessa variável. No entanto, devemos ser cautelosos ao fazer generalizações dos resultados para outros grupos socioeconômicos, pois os dados, obtidos por autorrelato, podem ter sido influenciados por essa variável contextual.

A idade dos sujeitos da amostra teve um papel importante em algumas das variáveis psicológicas por nós estudadas. Aliás, segundo Pless e Stein (1996), os resultados das investigações devem enquadrar-se num contexto desenvolvimental, uma vez que raramente são independentes da idade.

No nosso grupo de adolescentes com Paralisia Cerebral, os fatores do autoconceito ("aspecto comportamental","ansiedade" e "satisfação / felicidade") mantêm com a idade uma relação significativa, negativa. Ou seja, nos indivíduos mais velhos, os valores do autoconceito diminuem. Esse dado parece coincidir com a bibliografia consultada que documenta que o autoconceito dos jovens tende a diminuir ao longo do tempo e que só depois da adolescência estabiliza (Ruble, 1983; Marsh, 1988; Marsh, 1989, citados por Vaughn \& Elbaum, 1999), acrescentando Vaughn (1992, citados por Vaughn \& Elbaum, 1999) que, no caso de jovens com problemas de desenvolvimento, o percurso é semelhante, apresentando, também, essas características.

O nosso grupo com Paralisia Cerebral apresentou, igualmente, uma associação significativa, dessa vez positiva, da idade com o "coping ativo". Isto é, as estratégias de confronto ativo aumentam com a idade. Barros (1999) fez referência à importância da flexibilização das estratégias de coping que se vai adquirindo com a idade (uma vez que, entre o coping ativo e o coping não ativo, não se pode considerar que uma delas seja mais eficaz que outra; o mais adequado será adequar a estratégia à situação). SeiffgeKrenke et al. (2001) referiram que as investigações têm mostrado que os adolescentes mais velhos são mais ativos, têm uma gama mais ampla de estratégias de coping e mais capacidade para verem os seus problemas através de múltiplas perspectivas.

Quando comparamos os sexos, masculino e feminino, relativamente a todas as variáveis psicológicas, verificámos que é nas dimensões de sintomas psicopatológicos, sobretudo nas relacionadas com a ansiedade, 
que encontramos as diferenças significativas. Em todas essas dimensões é o sexo feminino que apresenta níveis mais elevados de sintomatologia psicopatológica.

Essa superioridade feminina, dos valores de ansiedade, parece coincidir com a informação da literatura, uma vez que os estudos indicam que há uma clara superioridade na prevalência de problemas de ansiedade no sexo feminino (Fabião, 1998; Rapee, Wignall, Hudson, \& Schniering, 2000; Vaz Serra, 1980). Thompson e Raezer (1998) testemunham, também, essa diferença, dizendo que as moças manifestam mais sintomas de angústia, aflição e ansiedade do que os rapazes. Allgood,-Merten, Lewinshon e Hops (1990) encontraram diferenças significativas entre os sexos para a depressão, sendo essa mais frequente nas adolescentes.

Quando nos referimos a níveis mais elevados de depressão e de ansiedade, não podemos deixar de aludir à comorbilidade. Kovacs (1990, citado por Compas \& Hammen, 1996), numa revisão da literatura, concluiu que até $70 \%$ dos casos de depressão manifestavam também sintomatologia ansiosa e que $30 \%$ a $75 \%$ tinham diagnóstico de perturbação ansiosa. Kashani e Carlson (1987, citados por Compas \& Hammen, 1996) também encontraram que $75 \%$ dos adolescentes que tinham distúrbios depressivos possuíam igualmente distúrbios de ansiedade. As crianças com perturbações de ansiedade preenchem, muitas vezes, os critérios para o diagnóstico de depressão. Bernstein (1991, citado por Fonseca, 1998), verificou que $50 \%$ das crianças com recusa à escola também tinham depressão e Last (1992, citado por Fonseca, 1998), através de uma amostra clínica, obteve uma taxa de comorbilidade com a depressão de 29 a $49 \%$. No nosso estudo, verificamos também que existe uma correlação positiva, significativa entre depressão e ansiedade.

Ao compararmos os vários níveis de compromisso motor, avaliados pela Gross Motor Function Classification System for Cerebral Palsy (Palisano et al., 1997), quanto às variáveis psicológicas, concluímos que não existiam diferenças significativas. A esse propósito gostaríamos de salientar que o fato dos indivíduos da nossa mostra não se distribuírem uniformemente pelos 5 níveis de gravidade que esse sistema de classificação contempla (situando-se sobretudo nos graus de menor compromisso motor e originando grupos com um número muito reduzido de sujeitos), dificultou a nossa tarefa de análise dos dados. Pelo que recomendamos que esse objetivo de investigação seja retomado em futuros estudos que tenham amostras mais alargadas e representativas de todos os índices de gravidade e que utilizem também formas alternativas de avaliação da gravidade da patologia motora.

Consideramos, então, os vários tipos clínicos de Paralisia Cerebral, e estabelecemos comparações entre eles, quanto ao autoconceito, às estratégias de coping e à sintomatologia psicopatológica. Apenas se mostraram significativos os valores da nota global do autoconceito e do respectivo fa- 
tor "estatuto intelectual / escolar". As diferenças nessas variáveis acontecem quando comparamos os tipos clínicos de Paralisia Cerebral que podemos considerar extremos, em termos de gravidade: o espástico hemiparético e o disquinético. $O$ disquinético, enquanto quadro mais aparatoso, apresenta valores significativamente mais elevados de autoconceito do que o espástico hemiparético, habitualmente um quadro mais discreto. Essa diferença é, de certo modo, surpreendente, uma vez que é o quadro mais grave que apresenta resultados mais elevados de autoconceito. No entanto, os autores têm salientado que há um maior desconforto quando o indivíduo com Paralisia Cerebral tem dificuldade em distinguir se deve incluir-se num grupo de deficientes ou se pode identificar-se mais com indivíduos normais; sendo essa avaliação mais clara e menos estressante nos casos com maior compromisso motor. Para uma pessoa com uma deficiência ligeira, muito próxima do normal, torna-se mais perturbador, porque, por ser mais fácil disfarçar a sua deficiência, tem dificuldade em prever a reação de quem descobre o seu problema (Shakespeare, 1975).

No nosso estudo, o autoconceito manteve, no geral, relações significativas, negativas com quase todas as dimensões de psicopatologia, avaliadas. O mesmo não se passou para as estratégias de coping.

Da revisão de estudos que fizemos acerca da relação do autoconceito com a psicopatologia, no geral, esses confirmam os nossos resultados. Vaz Serra, Antunes e Firmino (1986) encontraram que existe uma correlação negativa e significativa entre o autoconceito e sintomas depressivos, concluindo que quanto melhor for o autoconceito de um indivíduo, menos tendência ele terá para deprimir. Allgood-Merten, Lewinsohn e Hops (1990) encontraram também uma relação negativa entre autoestima e depressão, nos adolescentes normais.

Os resultados que obtivemos relativamente às dimensões do Inventário de Sintomas Psicopatológicos vêm confirmar os resultados de Mota Cardoso, Moura, Pais e Veríssimo (1986), numa investigação que efetuaram acerca da influência do autoconceito nas variáveis psicopatológicas. Com esse estudo, comprovaram também a relação entre um mau autoconceito e somatização, depressão, ansiedade e fobia, avaliados por um inventário de psicopatologia de nível neurótico (Middlesex Hospital Questionaire). Concluíram, então, que "o autoconceito é uma entidade vulnerabilizante dos síndromas psicopatológicos do foro neurótico" (Mota Cardoso et al., 1986, p. 191).

Parece-nos importante fazer referência à teoria de Bandura (1977, 1982), que salienta que uma autoavaliação positiva vai permitir ao sujeito ter um sentido de competência e eficácia pessoal, que é muito significativa para o seu bom funcionamento emocional, o que pode ajudar a explicar a correlação negativa significativa que encontramos entre o autoconceito e qualquer uma das escalas de avaliação da psicopatologia. 
Ainda relativamente ao autoconceito e à sua formação ao longo do desenvolvimento, julgamos ser também de realçar que o juízo que o indivíduo faz do próprio comportamento depende, em grande parte, das avaliações que lhe são feitas pelos outros (Vaz Serra, 1986).

Taylor, Asher e Williams (1987, citados por Vaugh \& Elbaum, 1999) referem que a aceitação dos pares tem uma grande importância no estatuto social do jovem e no seu autoconceito. Esses autores referem que se existe estigmatização, segregação e alienação é natural que o seu conceito de si seja mais pobre.

Por exemplo, se a criança com Paralisia Cerebral for continuamente criticada por ser desajeitada, pelos problemas motores e se for, frequentemente, chamada à atenção, essas avaliações, provavelmente, passarão a constituir um padrão de autoavaliação, nessa criança em desenvolvimento, que, com o passar do tempo, terá influência no seu autoconceito. Tendo em conta o modelo de Young dos esquemas precoces mal adaptativos, poderemos considerar, também, que sendo os esquemas desenvolvidos durante a infância e elaborados ao longo da vida, esse tipo de experiências vão ser fundamentais para a formação daquilo que serão os "filtros" para o processamento das experiências futuras. Os referidos"filtros" podem, nesse sentido, tornar-se disfuncionais, uma vez que as vivências dessas crianças envolvem frequentemente uma grande dependência e situações repetidas de críticas acompanhadas de embaraço. Nessa sequência, essas vivências podem contribuir para a formação de esquemas disfuncionais, tais como "defeito/vergonha", "isolamento social/alienação", "dependência/incompetência"',fracasso", entre outros (Young, 1990, 1999).

É muito importante que sejam estudados em futuras investigações esse funcionamento esquemático e as experiências sociais dos indivíduos com Paralisia Cerebral.

Quanto ao estilo de coping, o "coping não ativo" foi aquele apresentou uma relação significativa com a sintomatologia psicopatológica. $\mathrm{O}^{\prime \prime} \mathrm{co}$ ping ativo" não mostrou associações significativas com psicopatologia.

No "coping não ativo", as associações são negativas, apresentando-se significativas no caso da nota total de ansiedade, na "ansiedade global", e nos índices do BSI: IGS, "somatização"," "depressão,"'ansiedade,"“hostilidade" e "psicoticismo".

Segundo Vaz Serra, Ramalheira e Firmino (1988), os indivíduos com perturbações emocionais têm mecanismos de coping mais pobres, o que está de acordo com os nossos resultados.

Contudo, há tendência para se considerar as estratégias mais abertamente ativas e concretas como mais eficazes, revelando alguns estudos que essas se relacionam com um melhor ajustamento (Band, 1990; Matos, 1995; Peterson, 1989; Taylor \& Aspinwall, 1993).

Os estudos parecem indicar que as estratégias de confronto mais passivo estão mais relacionadas com a psicopatologia. 
Friedman (1990, citado por Stanton et al., 2000) e Carver (1993, citado por Stanton et al., 2000) afirmam que a utilização do coping de evitamento cognitivo e comportamental indicia mau ajustamento.

Um estudo de Knight et al. (1979) encontrou que as crianças com um estilo mais passivo de coping, que faziam evitamentos, apresentavam níveis mais elevados de ansiedade.

Asarnow (1987, citado por Herman-Stahl \& Petersen, 1996) e Garber (1991, citado por Herman-Stahl \& Petersen, 1996) referiram também que as crianças deprimidas adotam mais estratégias de evitamento e formas menos adequadas de ultrapassar o humor negativo.

Seiffge-Krenke (1993) encontrou que adolescentes diagnosticados com depressão, ansiedade e outros problemas psicopatológicos apresentavam níveis mais elevados de "coping por afastamento". Mais recentemente, Seiffge-Krenke e Klessinger (2000) obtiveram que o"coping de evitamento" tem efeitos a longo prazo na sintomatologia depressiva, em adolescentes.

Contudo, o "coping não ativo" não contempla, somente, um afastamento ou demissão das situações, devido a uma abordagem mais fatalista, mas considera também as estratégias internas de coping, que lidam mais diretamente com a emoção. Segundo Folkman e Moskowitz (2004) um coping eficaz adequa-se às características do contexto em que o indivíduo está inserido e mais recentemente tem-se salientado os resultados positivos de estratégias focadas na emoção e de regulação emocional. Santon et al. (2000) encontraram que o coping emocional, que envolve processar ativamente e expressar as emoções, pode ser adaptativo a curto prazo, em mulheres com cancro da mama, mas que a longo prazo pode tornar-se ruminativo e associar-se com menor ajustamento.

Não podemos, então, afirmar, à partida, que o "coping não ativo" seja menos eficaz. Além disso temos que ter em conta que a Paralisia Cerebral, é uma condição "para a vida", irreversível e não-progressiva, com a qual os indivíduos nascem e crescem, havendo, por isso, características únicas que Ihe estão associadas e que vão certamente influenciar esse processo de adaptação a longo prazo.

Os nossos resultados merecem-nos ainda um comentário sobre a própria avaliação das estratégias de coping. Como sabemos, esse conceito é complexo e difícil de medir e pensamos que a versão portuguesa do CASQ que utilizamos, ao contemplar somente as duas dimensões, "coping ativo" e "coping não ativo" está misturando, nessa última dimensão, itens que na versão original pertencem a dois fatores distintos ("coping interno" e "afastamento") o que não nos parece muito adequado e pode ter influenciado os resultados. Pensamos que poderá ser importante, em futuros estudos, ter esse aspecto em conta na avaliação do coping e usar até outras formas de avaliar esse conceito, por exemplo recorrer à metodologia de entrevista, pois embora seja mais morosa pode dar-nos informações que são difíceis de colher sob a forma de questionários estruturados de autorresposta. 
Para finalizar gostaríamos de salientar as implicações que os nossos resultados podem ter para a prevenção e tratamento da sintomatologia psicopatológica nos adolescentes com Paralisia Cerebral. Podemos concluir que estratégias de intervenção psicológica voltadas para a promoção de estratégias de coping interno de regulação emocional e de um bom autoconceito podem contribuir para melhorar a saúde mental desses jovens. Não queremos deixar de sublinhar também algumas limitações do nosso estudo, como o fato de ter um desenho transversal e de ter estudado apenas adolescentes com uma capacidade intelectual normal, o que nos impossibilita de fazer inferências causais e de generalizar os resultados para toda a população de adolescentes com Paralisia Cerebral.

\title{
Cerebral palsy in adolescence: results from a research
}

\begin{abstract}
It is very important to study self-concept, coping and mental health in adolescents suffering from Cerebral Palsy. This population has been rarely studied. Besides, these variables can contribute to the adaptation of these youth that face very adverse conditions, and can be protective and risk factors to their psychological health. We studied a sample of 78 subjects suffering from Cerebral Palsy (aged between 12 and 19 years old).We found the following results:a) girls show higher levels of psychopathology than boys, b) with age, the values of self-concept are lower and the values of "non active coping" are higher, c) some types of diagnosis of Cerebral Palsy differentiate themselves by the self-concept, $d$ ) self-concept correlates negatively with all indexes of psychopathology, e) "non active coping" correlates negatively with anxiety and some indexes of psychopathological symptomatology from BSI.
\end{abstract}

Keywords: Adolescence. Cerebral palsy. Coping. Self-concept. Psychopathology.

\section{Paralysie cérébrale dans l'adolescence: résultats d'une recherche}

Résumé: C'est très important d'étudier le concept de soi, le coping et la santé mentale des adolescents souffrant de Paralysie Cérébrale. Cette population est très peu étudiée. Et ces variables peuvent contribuer pour l'adaptation de ces jeunes qui se confrontent avec des situations très adverses, pouvant être facteurs protecteurs ou de risque de leur santé psychologique. Nous avons étudié un échantillon de 78 sujets souffrant de Paralysie Cérébrale (âgés de 12 à 19 ans). On a trouvé les résultats suivants : $a$ ) les filles présentent des valeurs de psychopathologie plus hautes que les garçons ; b) au fur et à mesure que l'âge augmente les valeurs du concept de soi baissent et augmentent ceux du « coping non-actif »; c) il y a des différences entre 
certains types de diagnostique en ce qui concerne le concept de soi ; d) le concept de soi se corrélationne négativement avec tous les indexes de psychopathologie ; e) le « coping non-actif » se corrélationne négativement avec l'anxiété et certains indexes de symptomatologie du BSI.

Mots-clés: Adolescence. Paralysie cérébrale. Coping. Concept de soi. Psychopathologie.

\section{La parálisis cerebral en la adolescencia: resultados de una investigación}

Resumen: Es de primordial importancia estudiar el autoconcepto, el coping y la salud mental de adolescentes con Parálisis Cerebral. Esta población ha sido muy poco estudiada. Además, estas variables pueden contribuir para la adaptación de estes jóvenes que se enfrentan con situaciones más adversas, podiendo ser factores protectores y de riesgo de su salud psicológica. Estudiamos una muestra de 78 sujetos con Parálisis Cerebral (edades entre los 12 y 18 años). Encontramos los siguientes resultados: a) las mujeres presentan valores de psicopatología más elevados que los hombres; b) a la medida que aumenta la edad disminuyen los valores de auto-concepto y aumentan los del "coping no activo"; c) algunos tipos de diagnóstico de Parálisis Cerebral se diferencian por el auto-concepto; d) el auto-concepto se correlaciona negativamente con todos los índices de psicopatología; e) el "coping no activo" se correlaciona negativamente con la ansiedad y con algunos índices de sintomatología psicopatológica do BSI.

Palabras-clave: Adolescencia. Parálisis cerebral. Coping. Auto-concepto. Psicopatología.

\section{Referências}

Alexander, M. A. (1990). Cerebral palsy. In M. Hersen \& V. B. Van Hasselt (Eds.), Psychological aspects of developmental and physical disabilities (pp. 87-93). California: Sage.

Allgood-Merten, B., Lewinsohn, P. M., \& Hops, H. (1990). Sex differences and adolescent depression. Journal of Abnormal Psychology, 99(1), 55-63.

Andrada, M. G. (1986). Cerebral palsy: Issues in incidence, early detection, and habilitation in Portugal. In K. Marfo, S. Walkek \& B. Charles (Eds.), Childhood disability in developing countries - Issues in rehabilitation and special education (pp. 72-80). New York: Praeger. 
Band, E. (1990). Children's coping with diabetes: Understanding the role of cognitive development. Journal of Pediatric Psychology, 15, 27-41.

Bandura, A. (1977). Self-efficacy: Toward a unifying theory of behavioural change. Psychological Review, 84, 191-215.

Bandura, A. (1982). Self-efficacy mechanism in human agency. Americam Psychologist, 35, 122-147.

Barros, L. (1999). Psicologia pediátrica - Perspectiva desenvolvimentista. Lisboa: Climepsi.

Bradford, R. (1997). Children, families and chronic disease: Psychological models and methods of care. New York: Routledge.

Canavarro, M. C. (1999). Inventário de sintomas psicológicos - BSI. In M. R. Simões, L. S. Almeida \& M. M. Gonçalves (Eds.), Testes e provas psicológicas em Portugal (Vol. 2, pp. 95-109). Braga: APPORT/SHO.

Cleto, P. M. (1998). Adaptação à mudança de escola no início da adolescência. Dissertação de Mestrado, Faculdade de Psicologia e de Ciências da Educação, Universidade do Porto, Portugal.

Compas, B. E., \& Hammen, C. L. (1996). Child and adolescent depression: Covariation and comorbidity in development. In R. J. Haggerty, L. R. Sherrod, N. Garmezy \& L. Rutter (Eds.), Stress, risk and resilience in children and adolescents (pp. 225-267). Cambridge: Cambridge University Press.

Derogatis, L. R. (1982). Self-report measures of stress. In L. Goldberger \& S. Brenznitz (Eds.), Handbook of stress. New York: Free.

Fabião, E. (1998). Ansiedade, aborrecimento, depressão e tentativa de suicídio. Psiquiatria Clínica, 19(2), 129-137.

Florian, V., \& Findler, L. (2001). Mental health and marital adaptation among mothers of children with cerebral palsy. American Journal of Orthpsychiatry, 71(3), 358-367.

Folkman, S., \& Moskowitz, J. T. (2004). Coping: Pitfalls and promise. Annual Reviews Psychology, 55, 745-774.

Fonseca, A. C. (1992). Uma escala de ansiedade para crianças e adolescentes: o que eu penso e o que eu sinto. Revista Portuguesa de Pedagogia, 26(1), 141-155.

Fonseca, A. C. (1998). Problemas de ansiedade em crianças e adolescentes: elementos para a sua caracterização e para o diagnóstico. Revista Portuguesa de Pedagogia, $33(2), 7-34$.

Harris, J. (1998). Cerebral palsy. In P. Howlin (Ed.), Behavioural approaches to problems in childhood. London: Cambridge University Press.

Joyce-Moniz, L., \& Barros, L. (2005). Psicologia da doença para cuidados de saúde. Porto: Asa.

Herman-Stahl, M., \& Petersen, A. C. (1996). The protective role of coping and social resources for depressive symptoms among young adolescents. Journal of Youth and Adolescence, 25(6), 733-753.

Knight, R., Atkins, A., Eagle, C., Evans, N., Finkelstein, J., Furkushima, D., et al. (1979). Psychological stress, ego defences, and cortisol production in children hospitalized for elective surgery. Psychosomatic Medicine, 41, 40-49. 
Koman, L. A., Smith, B. P., \& Shilt, J. S. (2004). Cerebral palsy. The Lancet, 363(9421), 1619-1630.

Kovacs, M. (1983). The children's depression inventory: A self-depression scale for schoolaged youngsters. University of Pittsburg, School of Medicine. Unpublished manuscript.

Lazarus, R. S., \& Folkman, S. (1984). Stress, appraisal, and coping. New York: Springer.

Livneh, H., \& Antonak, R. F. (2005). Psychosocial adaptation to chronic illness and Disability. A primer for counsellors. Journal of Counselling and Development, 83(1), 12-20.

Mann, M., Hosman, C. M., Schaalma, H. P., \& Vries, N. K. (2004). Self-esteem in a broadspectrum approach for mental health promotion. Health Education Research, 19(4), 357-372.

Marques, J. H. F. (1970). Manual da Escala de Inteligência de Wechsler para Crianças (WISC) - adaptação e aferição para Portugal. Lisboa: Instituto da Alta Cultura - Centro de Estudos de Psicologia e de História da Filosofia anexo à Faculdade de Letras da Universidade de Lisboa.

Matos, A. P. S. (1995). Factores psicológicos na doença coronária. Dissertação de Doutoramento em Psicologia Clínica, Faculdade de Psicologia e Ciências da Educação da Universidade de Coimbra, Portugal.

Miller, G., \& Clark, G. D. (1998). The cerebral palsy: Causes, consequences and management. Boston: Butterworth-Heinemann.

Mota Cardoso, R., Moura, L., Pais, A. B., \& Veríssimo, R. F. B. (1986). O autoconceito e as perturbações psicopatológicas de nível neurótico. Psiquiatria Clínica, 7(3), 189193.

Palisano, R., Rosenbaum, P., Walter, S., Russell, D., Wood, E., \& Galuppi, B. (1997). Gross motorfunctionclassification systemforcerebralpalsy.Canada:Neurodevelopmental Clinical Research Unit, McMaster University, Faculty of Health Sciences.

Peterson, L. (1989). Coping by children undergoing stressful medical procedures: Some conceptual, methodological and therapeutic issues. Journal of Consulting and Clinical Psychology, 57, 380-387.

Piers, E. V. (1984). Piers-Harris children's self-concept scale. Revised Manual. Los Angeles: Western Psychological Services.

Pless, I. B., \& Stein, R. E. K. (1996). Intervention research: Lessons from research on children with chronic disorders. In R. J. Haggerty, L. R. Sherrod, N. Garmezy \& M. Rutter (Eds.), Stress, risk and resilience in children and adolescents (pp. 317-353). Cambridge: Cambridge University Press.

Rapee, R. M., Wignall, A., Hudson, J. L., \& Schniering, C. A. (2000). Treating anxiety disorders - An evidence-based approach. Canada: New Harbinger.

Reynolds, C. R., \& Richmond, B. O. (1978). What I think and feel: Revised measure of children manifest anxiety. Journal of Abnormal Child Psychology, 6, 271-280.

Russman, B. (1991). Introduction. In J. R. Gage (Ed.), Gait analysis in cerebral palsy. London: MacKeith Press. 
Salmon, P. (2000). Psychology of medicine and surgery. A guide for psychologists, counsellors, nurses and doctors. Chichester: Wiley.

Sedas Nunes, A., \& Miranda, D. (1970). A composição social da população portuguesa: alguns aspectos e implicações. Revista Análise Social, (27), 28.

Seiffge-Krenke, I. (1993). Coping behavior in normal and clinical samples: More similarities than differences? Journal of Adolescence, 16, 285-304.

Seiffge-Krenke, I. (1998). Chronic disease and perceived developmental progression in adolescence. Developmental Psychology, 34(5), 1073-1084.

Seiffge-Krenke, I., \& Shulman, S. (1990). Coping style in adolescence. Journal of CrossCultural Psychology, 21(3), 351-377.

Seiffge-Krenke, I., \& Klessinger, N. (2000). Long-term effects of avoidant coping on adolescents' depressive symptoms. Journal of Youth and Adolescence, 3, 325-342.

Seiffge-Krenke, I., Weidemann, S., Fentner, S., Aegenheister, N., \& Poeblau, M. (2001). Coping with school-related stress and family stress in healthy and clinically referred adolescents. European Psychologist, 6(2), 123-132.

Shakespeare, R. (1975). Psychological effects of handicap - Essential psychology. London: Methuen.

Simões, M. R. (1999). A depressão em crianças e adolescentes: elementos para a sua avaliação e diagnóstico. Psychologica, 21, 27-64.

Simões, M., \& Albuquerque, M. C. (1989). Avaliação psicológica da criança e do adolescente: escalas, questionários e inventários. (Colecção textos de apoio, do Serviço de Defectologia da Faculdade de Psicologia e de Ciências da Educação da Universidade de Coimbra, série A: testes, n. 9, 18-25).

Stanton, A., Danoff-Burg., S., Cameron, C., Bishop, M., Collins, C., Kirk, S. B. et al. (2000). Emotionally expressive coping predicts psychological and physical adjustment to breast cancer. Journal of Consulting and Clinical Psychology, 68(5), 875-882.

Sukumaran, S., Vickers, B., Yates, P., \& Garralda, M. E. (2003). Self-esteem in child and adolescent psychiatric patients. European Child \& Adolescent Psychiatry, 12, 190197.

Taylor, S. E. (1991). Health psychology. New York: McGraw-Hill.

Taylor, S. E., \& Aspinwall, L. G. (1993). Coping with chronic illness. In L. Goldberger, \& S. Breznitz (Eds.), Handbook of stress, theoretical and clinical aspects (2nd ed., pp. 511-531). New York: Free.

Thompson, R. J., \& Gustafson, K. (1996). Adaptation to chronic childhood Disease. Washington, DC: American Psychological Association.

Thompson, R. J., \& Raezer, L. B. (1998). Families of children with chronic medical conditions. In A. S. Bellack \& M. Hersen (Eds.), Comprehensive clinical psychologyapplication in disease populations (Vol. 9). USA: Pergamon.

Vaughn, S., \& Elbaum, B. (1999). The self-concept and friendships of students with learning disabilities: A developmental perspective. In R. Gallimore, L. Bernheimer, D. L. MacMillan, D. L. Speece \& S. Vaughn (Eds.), Developmental perspective on children with high-incidence disabilities. Mahwah, NJ: Lawrence Erlbaum. 
Vaz Serra, A. (1980). O que é a ansiedade? Psiquiatria Clínica, 7(2), 93-104.

Vaz Serra, A. (1986). A importância do autoconceito. Psiquiatria Clínica, 7(2), 57-66.

Vaz Serra, A., Antunes, R., \& Firmino, H. (1986). Relação entre autoconceito e expectativas. Psiquiatria Clínica, 7(2), 85-90.

Vaz Serra, A., Ramalheira, C., \& Firmino, H. (1988). Mecanismos de coping: diferenças entre população normal e doentes com perturbações emocionais. Psiquiatria Clínica, 9(4), 323-328.

Veiga, F. H. (1989). Escala de autoconceito: adaptação portuguesa do "Piers-Harris Children's Self-Concept Scale". Psicologia, 7(3), 275-284.

Young, J. E. (1990). Cognitive therapy for personality disorders: A schema-focused approach. Sarasota, FL: Professional Resource Exchange.

Young, J. E. (1999). Cognitive therapy for personality disorders: A schema-focused approach (3rd ed.). Sarasota, FL: Professional Resource Press.

Wallander, J. L., Varni, J. W., Babani, L., Banis, H. T., \& Wilcox, K. T. (1989). Familiy resources as resistance factors for psychological maladjustment in chronically ill and handicapped children. Journal of Pediatric Psychology, 14, 157-173.

Ana Paula Matos, Doutora em Psicologia Clínica, Professora da Faculdade de Psicologia e de Ciências da Educação da Universidade de Coimbra. Endereço para correspondência: Rua do Colégio Novo, apartado 6153, 3001-802, Coimbra. Endereço eletrônico: apmatos@fpce.uc.pt

Joana Castela Lobo, Mestre em Psicologia Clínica, Psicóloga da Associação de Paralisia Cerebral (APPC-NRC), Centro de Reabilitação da Paralisia Cerebral de Coimbra. Endereço eletrônico: jcastelalobo@hotmail.com

Recebido em: 7/09/2008

Aceito em: 2/11/2008 
